\title{
Influence of magnetic interactions on the Anisotropy of Magnetic Susceptibility: The case of single domain particles packed in globule aggregates
}

\author{
G. Fanjat*, V. Shcherbakov ${ }^{\dagger}$ P. Camps ${ }^{\ddagger}$
}

\begin{abstract}
The influence of magnetic interactions on the anisotropy of magnetic susceptibility (AMS) have been largely studied by several theoretical models or experiments. Numerical models have shown that when magnetostatic interactions occur, the distributions of particles over the volume rather than their individual orientations control the AMS. We have shown recently from a comprehensive rock magnetic study and from a theoretical 2-dimensional (2-D) model that single domain particles closely packed in globule aggregate could produce strong local random interaction magnetic fields which could influence the magnetic susceptibility and decrease the degree of anisotropy. In this paper, we first present in detail this 2-D theoretical model and then we extend it to the $3-\mathrm{D}$ case. The possible distribution function of the magnetostatic interaction fields comprises two extreme states: it is either isotropic or ordered. The former case corresponds to the thermal-demagnetized state while the second case corresponds to the Alternative Field (AF) demagnetized state. We show that when easy axes of magnetization are not uniformly distributed, the degree of anisotropy decreases as the interaction field increases in both AF- and thermal-demagnetized states in 2-D and 3-D geometry. Thus we conclude that random magnetic fields generated by a random arrangement of magnetic particles over the sample volume decrease the degree of anisotropy of AMS and may alter the magnetic fabric.
\end{abstract}

\section{Introduction}

Measurement of the low-field anisotropy of magnetic susceptibility (AMS) in rocks provides a second-order tensor represented by an ellipsoid whose principal axes, $K_{1}>K_{2}>K_{3}$, are determined by the contribution of the individual minerals (Tarling and Hrouda, 1993, see e. g.). Such AMS measurements are commonly used as a proxy of deformation in petrofabric and structural studies (e.g. Borradaile and Jackson, 2004, for a review). Easy, rapid and inexpensive to implement, this method as been successfully calibrated to practically every rock including those weakly deformed such as lava flows (Cañón-Tapia, 2004) or magma flows (Bouchez, 2000). An expected model is a normal AMS fabric corresponding to a preferred orientation of non-equant-shaped magnetic grains. In this basic model, the maximum axes $K_{1}$ are aligned along the long axes of the grains and thus are statistically parallel to the flow direction while the minimum axes $K_{3}$ are perpendicular to the flow plane. Numerical models have been developed to determine the relationship between the orientation distribution of magnetic minerals and AMS (Jezek and Hrouda, 2004). However, these relationships can be complicated by different magnetic configurations or particular strains (Merle, 1998). Potter and Stephenson (1988) and Rochette et al. (1999) have shown situations where inverse magnetic fabrics are due to a predominance of SD grains ( $K_{1}$ perpendicular to the flow plane and $K_{3}$ parallel to the flow direction) and Rochette et al. (1999) gave examples where the magnetic fabric is intermediate between the normal and inverse models. These different patterns depend on the physical origin of the AMS. It is clear that interpretations of flow directions from magnetic fabric data are

\footnotetext{
* Géosciences Montpellier, CNRS and University of Montpellier, France

$\dagger^{\dagger}$ Borok Geophysical Observatory, 152742 Borok, Nekouz, Yaroslavl region, RF, Russia.

${ }^{\ddagger}$ Géosciences Montpellier, CNRS and University of Montpellier, France
} 


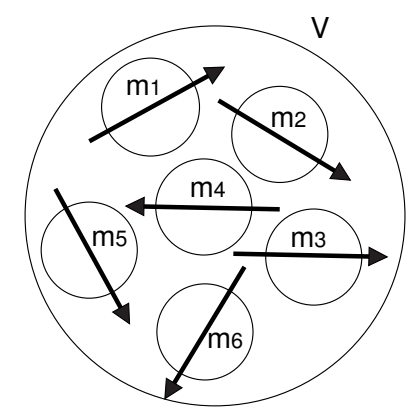

Figure 1: 2-D schematic representation of magnetic particles in a cluster of a finite volume $V$. Each particle own a magnetic moment $\mathbf{m}_{\mathbf{i}}$ producing a dipolar magnetic field interacting with the field produced by the neighboring particles.

not as straightforward as usually assumed. All these studies pointed out the main role of distribution and magnetic behaviour of the particles. Indeed, distribution anisotropy is a common, if not ubiquitous, source of AMS in weakly deformed rocks such as basaltic lava flows. It occurs when equant- or not-equant-shaped magnetic particles are unevenly distributed and are close enough to interact magnetostatically, producing an asymmetric magnetic interaction field which contributes to the bulk magnetic anisotropy (Hargraves et al., 1991). The role of such magnetostatic interactions on magnetic susceptibility have been largely studied and several theoretical models have been developed. Stephenson (1994) showed that magnetically isotropic particles with linear or planar regular arrangements give rise to large degree of anisotropy. Cañón-Tapia (1996) and Gaillot et al. (2006) generalized the latter models by studying the effects of individual-particle anisotropy in AMS. These models have shown that when magnetostatic interactions occur, the distributions of particles rather than their individual orientations controls the AMS. Bascou et al. (2005) were the first to demonstrate experimentally the predominance of the distribution anisotropy in basaltic lava flow by comparing the magnetic fabric with the lattice preferred orientations of plagioclase, clinopyroxene and titanomagnetite. However Grégoire et al. (1998) concluded on the basis of experimental results that shape orientation of magnetite grains seems to be the main source of AMS even in case when magnetic interactions are strong. Moreover, Grégoire et al. (1995) showed that the bulk susceptibility and the degree of anisotropy change as a function of grain separation and grain configuration in a system formed by two ferromagnetic particles. In a recent study Fanjat et al. (2011) concluded from a comprehensive rock magnetic study and from a 2-D theoretical model that uniaxial anisotropic SD grains randomly distributed over the volume of a cluster could produce random interaction magnetic fields which could decrease the degree of anisotropy and thus alter the magnetic fabric. The purpose of the present study is to present in detail the 2-D model and to develop it in a 3-D geometry. This paper is organized as follows. We present in a first section the outlines of our model which is then developed in a 2-D case and a 3-D case, respectively. The last section contains concluding comments on the theory and results.

\section{Numerical approach}

The direct way to model systems of interacting particles is to introduce a local interaction field $H_{\text {int }}$. For a given grain, $H_{\text {int }}$ is produced by all other grains in the cluster (Fig. 1). Underline that magnetic particles are randomly distributed over the cluster volume $V$, the local interaction fields are random and can be described statistically with a probability density function $W\left(H_{\text {int }}\right)$. Moreover, the closer are the particles and the stronger are their mutual interactions. To account for this effect, we used the packing fraction $p$ (Shcherbakov and Shcherbakova, 1975; Egli, 2006b). Consider an ensemble of SD spheres closely packed, $p$ is defined by the ratio between the volume occupied by the magnetic particles and the total volume $V$ of the cluster. The size for SD magnetite grains is 0.03-0.05 $\mu \mathrm{m}$ (Dunlop and Özdemir, 1997). Moreover, numerical simulations show that 
the maximal packing fraction for random close packing spheres is 0.636 (Torquato et al., 2000). According to the cluster sizes observed in basaltic flows (Zhou et al., 1997; Fanjat et al., 2011) and the size of SD particles, we assume that the clusters are composed by two scales of packing spheres: SD particles are gathered in small cluster of about $0.1 \mu \mathrm{m}$, itself packed in a bigger one. In this case, the maximal packing fraction is evaluated between 0.20 and 0.40 , in agreement with values proposed by Chen et al. (2007) in natural and biogenic cluster of SD particles. For such densely packed clusters, the distribution function $W\left(H_{\text {int }}\right)$ can be approximated by a Gaussian distribution (Shcherbakov and Shcherbakova, 1975; Egli, 2006a):

$$
W\left(H_{\text {int }}\right)=\frac{1}{\sigma_{0} \sqrt{2 \pi}} \exp \left(-\frac{H_{\text {int }}^{2}}{2 \sigma_{0}^{2}}\right) .
$$

Here the standard deviation $\sigma_{0}$ is defined by:

$$
\sigma_{0}=0.3 \mu_{s} \sqrt{p}
$$

where $\mu_{s}$ denotes the intensity of the saturation magnetization. For magnetite, $\mu_{s} \approx 480 \mathrm{kA} / \mathrm{m}$ at $p \approx(0.2-0.4)$, thus the dispersion $\sigma_{0}$ achieves values of order of $80 \mathrm{kA} / \mathrm{m}$. This means that the local fields can be relatively strong.

\section{$3 \quad 2-\mathrm{D}$ geometry}

\subsection{Magnetic energies for SD particles with uniaxial anisotropy}

First, let us consider an ensemble of identical oriented spherical particles. For simplicity, we will assume grains with an uniaxial anisotropy constant $K$. Let us suggest in this section that all the vectors, i.e. spontaneous magnetization $\mathbf{M s}$, interaction field $\mathbf{H}_{\mathbf{i n t}}$, and small external field $\mathbf{h}_{\mathbf{e x t}}$, lie in the same plane (Fig. 2). Thus we can use a 2-D geometry for the further calculations and introduce a polar coordinate system with the polar axis defined by the unit vector $\mathbf{u}$ along the easy axis of magnetization 1 . In this frame, the vectors have the following coordinates:

$$
\mathbf{M}_{\mathbf{s}}=M_{s}\left(\begin{array}{c}
\sin \theta \\
\cos \theta
\end{array}\right) \quad \mathbf{H}_{\mathbf{i n t}}=H_{\text {int }}\left(\begin{array}{c}
\sin \phi \\
\cos \phi
\end{array}\right) \quad \mathbf{h}_{\mathbf{e x t}}=h_{\text {ext }}\left(\begin{array}{c}
\sin \psi \\
\cos \psi
\end{array}\right)
$$

In absence of external and interaction magnetic fields, $\mathbf{M}_{\mathbf{s}}$ lies along $\mathbf{l}$. In presence of an internal interaction magnetic field $\mathbf{H}_{\mathbf{i n t}}, \mathbf{M}_{\mathbf{s}}$ is shifted by an angle $\theta$ from its initial position (Fig. 2.a). The expression of the total non perturbed energy $E_{n p}$ of the particle is (see e.g. O'Reilly (1984)):

$$
E_{n p}=E_{a n}+E_{i n t}
$$

where

$$
E_{a n}=K \sin ^{2} \theta
$$

denotes the anisotropy energy of the uniaxial grain, and $K$ denotes the anisotropy parameter $(N a-$ gata, 1961) and

$$
E_{\text {int }}=-\mathbf{M}_{\mathbf{s}} \cdot \mathbf{H}_{\mathbf{i n t}}=-M_{s} H_{\text {int }} \cos (\theta-\phi)
$$

is the magnetostatic interaction energy. However, external fields are present in the general case, so the total energy density of the grain becomes:

$$
E=K \sin ^{2}(\theta)-M_{s} H_{\text {int }} \cos (\theta-\phi)-M_{s} h_{\text {ext }} \cos (\theta-\psi) .
$$

Here $\mathbf{h}_{\text {ext }}$ denotes a small external magnetic field and $\psi \in[-\pi ; \pi]$ denotes the angle between $\mathbf{h}_{\text {ext }}$ and $\mathbf{l}$ (Fig. 2.b). Under the influence of $\mathbf{h}_{\mathbf{e x t}}, \mathbf{M}_{\mathbf{s}}$ is slightly deflected from $\theta$ to $\theta^{\prime}$. Normalizing eq. 

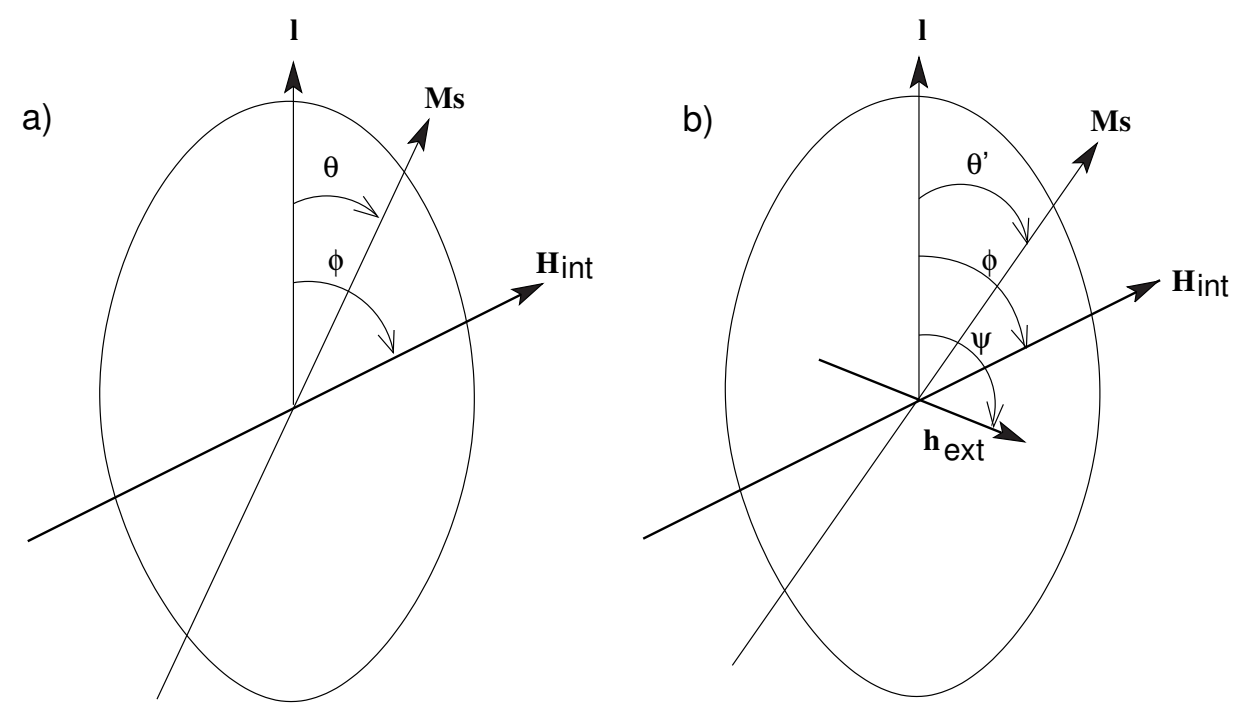

Figure 2: Sketch of a SD particle with uniaxial anisotropy: a) Submitted to an interaction magnetic field $\mathbf{H}_{\mathbf{i n t}} \cdot \mathbf{M}_{\mathbf{s}}$ is the spontaneous magnetization and $\phi$ is the angle between $\mathbf{H}_{\mathbf{i n t}}$ and the easy axis of magnetization $\mathbf{l}$. $\theta$ is the angle between the spontaneous magnetization $\mathbf{M}_{\mathbf{s}}$ and $\mathbf{l}$. b) Application of an external field $\mathbf{h}_{\text {ext }}$. $\psi$ is the angle between $\mathbf{h}_{\text {ext }}$ and $\mathbf{l}$. $\theta^{\prime}$ is the new angle of equilibrium of the spontaneous magnetization $\mathbf{M}_{\mathbf{s}}$.

(6) to $K$ and defining dimensionless interaction magnetic field $H=M_{s} H_{\text {int }} / K$ and dimensionless external magnetic field $h=M_{s} h_{\text {ext }} / K$, we find:

$$
E_{t o t}=\sin ^{2}(\theta)-H \cos (\theta-\phi)-h \cos (\theta-\psi)=E_{0}+\Delta E .
$$

where $E_{0}$ is the dimensionless non-perturbed energy density:

$$
E_{0}=\sin ^{2} \theta-H \cos (\phi-\theta)
$$

Underline that in this notation, the spontaneous magnetization of a particle is equal to one. The position of equilibrium $\theta_{0}$ ' of the magnetic moment is found by minimization of eq. (7). First, let us determine the extrema of $E_{0}$ in eq. (8). $\theta_{0}$ is a root of the following equation:

$$
\frac{\partial E_{0}}{\partial \theta}=\sin (2 \theta)-H \sin (\phi-\theta)=0 .
$$

Note that for $H<1$, there are always two minima states of the energy $E_{0}$ in the interval $[0, \pi]$ (Fig. 3.a). The left minimum is deeper at $\phi<\pi / 2$ while the right minimum is deeper at $\pi / 2<\phi<\pi$. These two minima correspond to two of the three possible roots of eq. (9). $\theta_{0}^{1}$ corresponds to $\phi<\pi / 2$ and $\theta_{0}^{2}$ corresponds to $\pi / 2<\phi<\pi$ (Fig. 3). However for $H>1$, the two minima exist at some $\phi$ only (Fig. 4.b). Keeping this result in mind, we consider only the case where $H<1$. This restriction in practice is of little importance because strong interaction fields with $H>1$ seldom occur, only at very high volume density of SD grains. Then, in the case when such very strong fields do appear, they would produce strong correlations between the direction of the magnetic moments of the grains and thus, the analysis presented hereafter will be anyway invalid.

Assuming smallness of $h$ with respect to $H$, the new position of equilibrium $\theta_{0}^{\prime}$ of eq. (7) is $\theta_{0}^{\prime}=\theta_{0}+y$ where $y \ll 1$. Expanding $E_{\text {tot }}$ in a Taylor's series at $\theta_{0}$, we obtain:

$$
E_{t o t}\left(\theta_{0}+y\right) \approx F\left[\theta_{0}(\phi)\right] \frac{y^{2}}{2}-h \cos \left(\psi-\theta_{0}-y\right)+E_{t o t}\left(\theta_{0}\right)
$$

where 


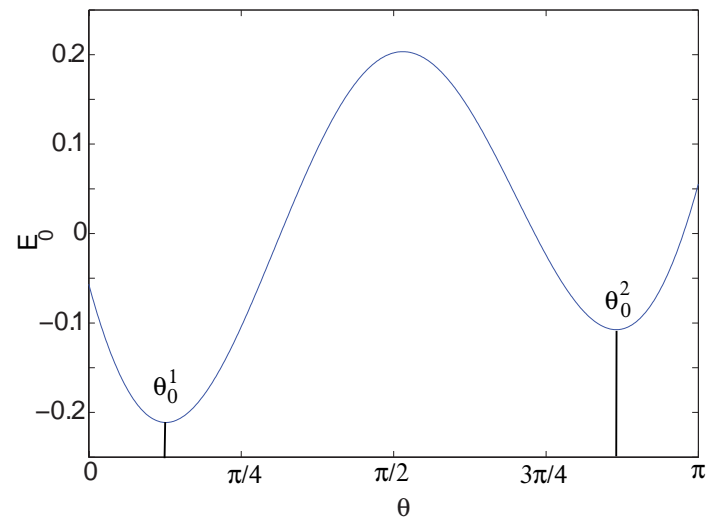

Figure 3: The non-perturbed energy density $E_{0}$ as a function of the angle $\theta$ for $H=0.8$ and $\phi=1.5$. The two minima are $\theta_{0}^{1}$ and $\theta_{0}^{2}$.

$$
F\left[\theta_{0}(\phi)\right]=\frac{\partial^{2} E_{0}}{\partial \theta^{2}}=2 \cos \left(2 \theta_{0}\right)+H \cos \left(\phi-\theta_{0}\right)
$$

is the second derivative of $E_{0}$. We obtain the expression of $y$ from eq. (10):

$$
\frac{\partial E_{t o t}}{\partial y}=F\left[\theta_{0}(\phi)\right] y-h \sin \left(\psi-\theta_{0}\right)=0,
$$

so

$$
y=h \frac{\sin \left(\psi-\theta_{0}\right)}{F\left[\theta_{0}(\phi)\right]} .
$$

The projection of the induced magnetization of a grain onto the field direction, is given for the two angles $\theta$ and $\psi$ by:

$$
m_{\text {ind }}(\psi)=h \frac{\sin ^{2}\left(\theta_{0}-\psi\right)}{F\left[\theta_{0}(\phi)\right]} .
$$

The total induced magnetic moment is obtained by the integration of eq. (14) over the surface. Now the further scenario depends on the distribution function of the easy axis of magnetization.

\subsection{Isotropic distribution for the easy axes}

Consider now a sample for which the distribution function for $\mathbf{H}_{\mathbf{i n t}}$ is isotropic. Then, introducing the blocking temperature $T_{b}$ of a grain, the probability that $\theta_{0}$ belongs to either $\theta_{0}^{1}$ or $\theta_{0}^{2}$ is described by the Boltzman's distribution:

$$
P\left[\theta_{0}^{i}(\phi)\right]=\frac{1}{C} \exp \left(-\frac{E_{0}\left[\theta_{0}^{i}(\phi)\right]}{k_{B} T_{b}}\right) \quad \text { for } \mathrm{i}=(1,2)
$$

where

$$
C=\exp \left(-\frac{E_{0}\left[\theta_{0}^{1}(\phi)\right]}{k_{B} T_{b}}\right)+\exp \left(-\frac{E_{0}\left[\theta_{0}^{2}(\phi)\right]}{k_{B} T_{b}}\right),
$$

and $k_{B}$ is the Boltzman's constant. From eq. (14), the total induced magnetic moment can be found by averaging: 
a)

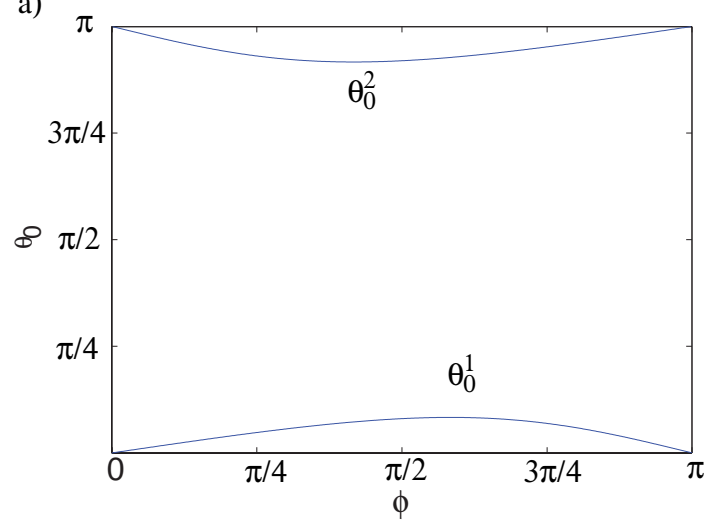

b)

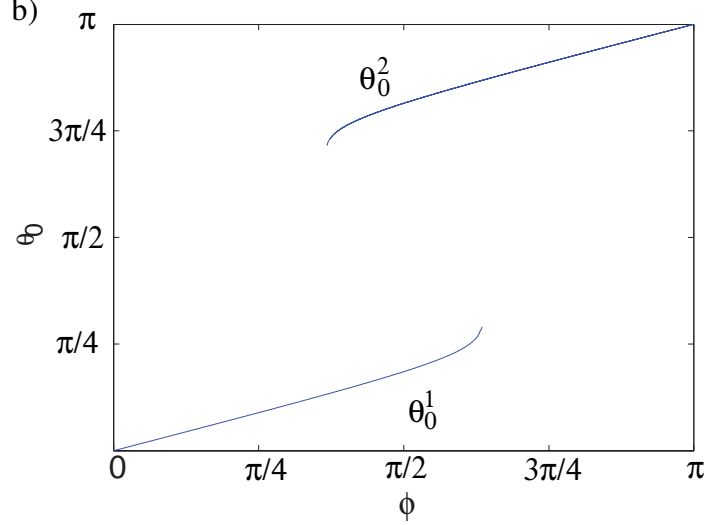

Figure 4: The positions of the minima $\theta_{0}^{1}$ and $\theta_{0}^{2}$ of the energy $E_{0}$ computed at the stable point $\theta_{0}$ as the function of the angle $\phi$ between the easy axis and direction of interaction field $H$. The figure is computed for a) $H=0.8$ and b) $H=1.1$.

$$
\begin{aligned}
M_{i n t}\left[\theta_{0}(\phi), \psi\right] & =\frac{1}{2 C \pi^{2}}\left(\int_{\frac{-\pi}{2}}^{\frac{\pi}{2}} \int_{-\pi}^{\pi}\left(\cos \left(\theta_{0}-\psi\right)+h \frac{\sin ^{2}\left(\theta_{0}-\psi\right)}{F\left[\theta_{0}(\phi)\right]}\right) f(\phi, \psi) d \psi \exp \left[-\frac{E_{0}^{1}(\phi)}{k_{B} T_{b}}\right] d \phi\right. \\
& +\int_{\frac{\pi}{2}}^{\pi} \int_{-\pi}^{\pi}\left(\cos \left(\theta_{0}-\psi\right)+h \frac{\sin ^{2}\left(\theta_{0}-\psi\right)}{F\left[\theta_{0}(\phi)\right]}\right) f(\phi, \psi) d \psi \exp \left[-\frac{E_{0}^{2}(\phi)}{k_{B} T_{b}}\right] d \phi \\
& \left.+\int_{-\pi}^{\frac{-\pi}{2}} \int_{-\pi}^{\pi}\left(\cos \left(\theta_{0}-\psi\right)+h \frac{\sin ^{2}\left(\theta_{0}-\psi\right)}{F\left[\theta_{0}(\phi)\right]}\right) f(\phi, \psi) d \psi \exp \left[-\frac{E_{0}^{2}(\phi)}{k_{B} T_{b}}\right] d \phi\right)
\end{aligned}
$$

where $f(\phi, \psi)$ denotes the distribution function of the angles $\phi$ and $\psi$. If there is no magnetic fabric, then $\psi$ has an uniform distribution over the interval $[0, \pi]$. Neglecting a possible correlation between the directions of magnetic moments of the grains, i.e. neglecting any anisotropy of distribution (Hargraves et al., 1991), the angle $\phi$ is also distributed uniformly over $[0, \pi]$. As a consequence, for the further estimations, we will assume that $f(\phi, \psi)=1$.

Whatever is the nature of the natural remanent magnetization (NRM) in a sample, the distribution function of the interaction field is comprised between two boundary states. It is either isotropic, which formally corresponds to the thermal-demagnetized state, or it is strongly ordered, which formally corresponds to the Alternative Field (AF) demagnetized state. To estimate the susceptibility measured from the thermal-demagnetized state, we consider the extreme case when the demagnetization completely randomizes the direction of the interaction field in relation to the position of easy axis of a grain. Physically, it happens when at a blocking temperature $T_{b}$, the interactions are weak, so the ratio $E_{0}^{i}(\phi) /\left(k_{B} T_{b}\right) \ll 1$. Then, all exponents in eq. (18) can be replaced by one and the factor $C$ equals 2 , so we obtain:

$$
M_{i n t}\left[\theta_{0}(\phi), \psi\right]=\frac{1}{4 \pi^{2}} \int_{-\pi}^{\pi} \int_{-\pi}^{\pi}\left(\cos \left(\theta_{0}-\psi\right)+h \frac{\sin ^{2}\left(\theta_{0}-\psi\right)}{F\left[\theta_{0}(\phi)\right]} d \psi\right) d \phi .
$$

Hence the integration over $\psi$ brings the first term in eq. (18) to zero. Thus, eq. (18) becomes:

$$
M_{i n t}\left[\theta_{0}(\phi), \psi\right]=\frac{h}{4 \pi^{2}} \int_{-\pi}^{\pi}\left(\int_{-\pi}^{\pi} \frac{\sin ^{2}\left(\theta_{0}-\psi\right)}{F\left[\theta_{0}(\phi)\right]} d \psi\right) d \phi
$$

After integrating eq. (19) over $\psi$, we obtain:

$$
M_{\text {int }}\left[\theta_{0}(\phi)\right]=\frac{h}{4 \pi} \int_{-\pi}^{\pi} \frac{d \phi}{2 \cos \left[2 \theta_{0}(\phi)\right]+H \cos \left[\theta_{0}(\phi)-\phi\right]}
$$


From eq. (20) we deduce the expression of the magnetic susceptibility:

$$
\chi_{T}(H)=\frac{1}{2 \pi} \int_{0}^{\pi} \frac{1}{2 \cos \left[2 \theta_{0}(\phi)\right]+H \cos \left[\theta_{0}(\phi)-\phi\right]} d \phi .
$$

The susceptibility of particles in thermal-demagnetized state increases when $H$ increases (Fig. 5). The reason for this increase is that the potential well becomes more shallow when $\pi / 2<\phi<\pi$, i.e. when the direction of the interaction field is more or less opposite to the easy axis of magnetization (Fig. 6). The sharp increase of the susceptibility at $H \rightarrow 1$ occurs because at this limit the second derivative $\mathrm{F}$ approaches zero at $\phi=3 \pi / 4$ (Fig 6 ). To show that, note first that when $H=1$ the analytical solution of eq. (9) can be calculated. Indeed, at $\phi<3 \pi / 4$, we have $2 \theta_{0}=-\phi+\theta_{0}$, so $\theta_{0}=\phi / 3$. Analogously, $\theta_{0}=\pi-\phi$ at $3 \pi / 4<\phi<\pi$. Hence, $F=3 \cos (2 \phi / 3)$ for $\phi<3 \pi / 4$ and $F=\cos (2 \phi)$ for $3 \pi / 4<\phi<\pi$, so

$$
\chi_{T}(H)=\frac{1}{6 \pi} \int_{0}^{3 \pi / 4} \frac{d \phi}{\cos (2 \phi / 3)}+\frac{1}{2 \pi} \int_{3 \pi / 4}^{\pi} \frac{d \phi}{\cos (2 \phi)} .
$$

It is straightforward to demonstrate that eq. (22) has a logarithmic divergence at $\phi \rightarrow 3 \pi / 4$.

Now let us consider the other extreme case. AF treatment forces the magnetic moment of a grain to switch from one to another direction along the easy axis. In the absence of any external or interaction field, it leads to a statistical polarization of the magnetic moments of SD ensemble when an half of them falls in one direction while the second half falls in the opposite one. However, the presence of any magnetic field breaks the symmetry, making these states not equiprobable. As far as the amplitude of AF gradually decreases, the moment finally will be trapped in the state with the biggest critical field, i.e. in the deepest potential well. Thus, even if the location of the magnetic moment before the $\mathrm{AF}$ treatment was in the shallow minimum $\theta_{0}=\theta_{0}^{2}$, it will occupy eventually the deep potential well $\theta_{0}=\theta_{0}^{1}$. Hence,

$$
\chi_{A F}(H)=\frac{1}{\pi} \int_{0}^{\frac{\pi}{2}} \frac{1}{2 \cos \left[2 \theta_{0}(\phi)\right]+H \cos \left[\theta_{0}(\phi)-\phi\right]} d \phi
$$

It is clearly demonstrated that the effect of interactions on the susceptibility of an ensemble of SD grains in AF-demagnetized state is not much important (Fig. 5). Indeed, the only result is that interactions slightly decrease the susceptibility which ranges now from 0.25 at $\mathrm{H}=0$ to 0.21 at $\mathrm{H}$ $=1$. Physically, the decrease of $\chi_{A F}$ with $H$ is conditioned by the deepening of the potential well of the states when $\phi<\pi / 2$, i.e. when the direction of the interaction field is close to the direction of the magnetic moment of a grain.

\subsection{Anisotropic distribution for the easy axes}

When a magnetic fabric is present, the easy axes are distributed non-homogeneously over the angles $\psi$. Hence the distribution function $f(\psi, \phi)$ is not constant. We assume for simplicity that the distribution of interaction field vector is totally random so that $f(\phi, \psi)=f(\psi)$. We can formally decompose $f(\psi)$ into Fourier series. Let us retain only the two first terms, so $f$ becomes:

$$
f(\phi, \psi)=1+a \cos (2 \psi),
$$

where $a$ denotes an arbitrary coefficient. With this choice, the maximum susceptibility axis lies along $\psi=0$.

For thermo-demagnetized state, using eq. (24), the expression of the total induced magnetic moment becomes:

$$
\begin{aligned}
M & =\frac{1}{4 \pi^{2}} \int_{-\pi}^{\pi} \int_{-\pi}^{\pi}\left(\cos \left(\theta_{0}-\psi\right)+h \frac{\sin ^{2}\left(\theta_{0}-\psi\right)}{F\left[\theta_{0}(\phi)\right]}\right)(1+a \cos (2 \psi)) d \phi d \psi \\
& =\frac{h}{8 \pi} \int_{-\pi}^{\pi} \frac{2-a \cos \left(2 \theta_{0}\right)}{F\left[\theta_{0}(\phi)\right]} d \phi
\end{aligned}
$$




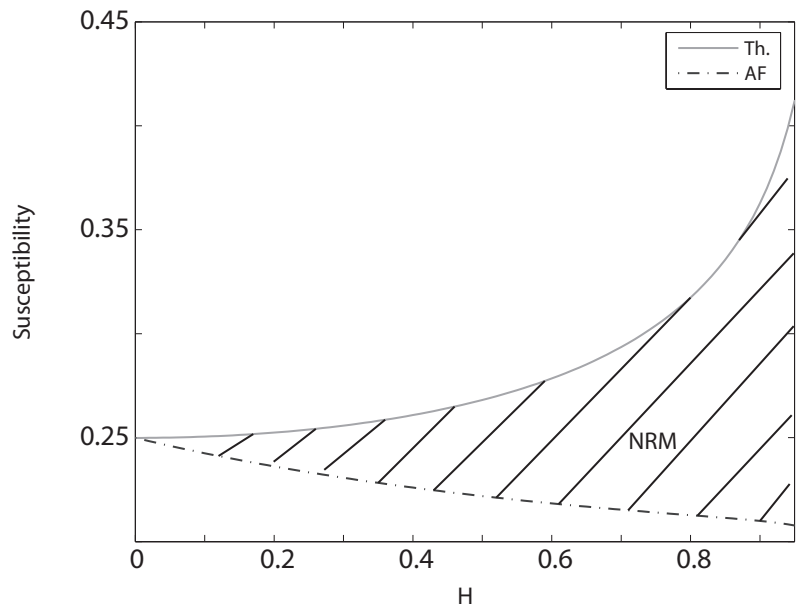

Figure 5: Influence of the interaction field on the bulk susceptibility in the 2-D case. Theoretical magnetic susceptibility of AF-demagnetized and thermo-demagnetized grain as a function of the interaction field $\mathrm{H}$. The shaded area is the area where the NRM is likely to be.

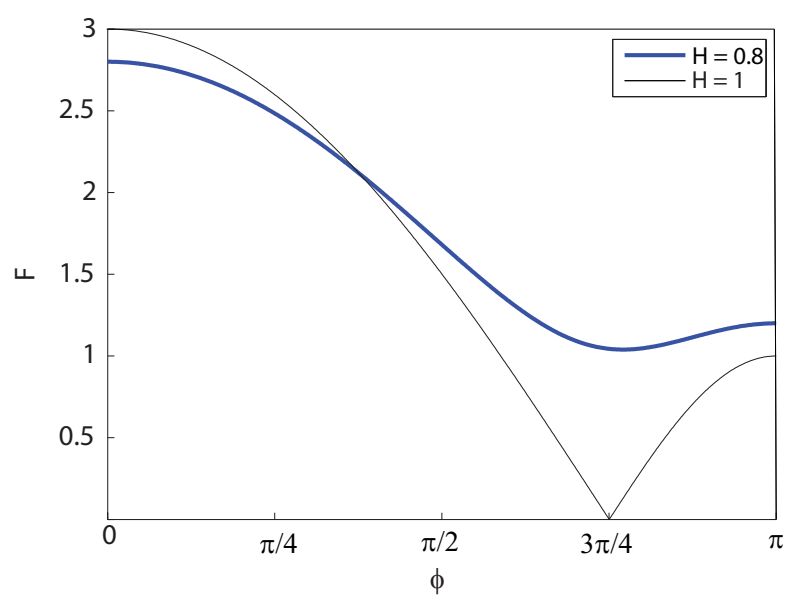

Figure 6: The second derivative $\mathrm{F}$ computed at the stable point $\theta_{0}$ as the function of the angle $\phi$. The thick curve is computed for $\mathrm{H}=0.5$ while the thin curve is computed for $\mathrm{H}=1.0$. 


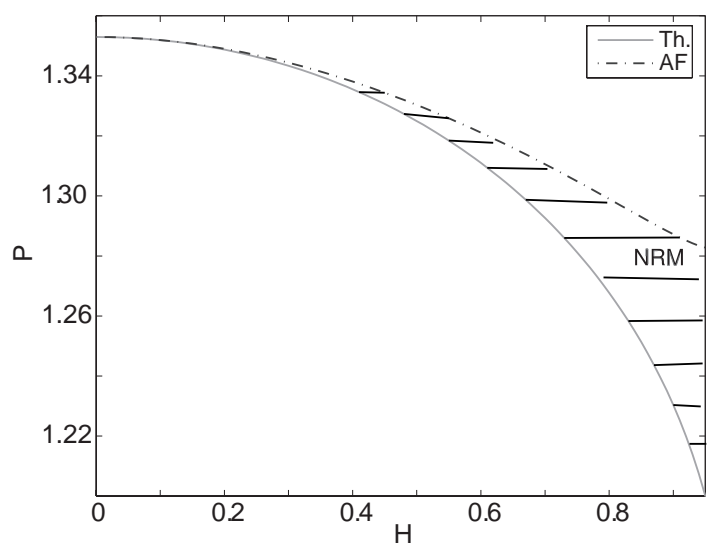

Figure 7: Influence of the interaction field on the anisotropy degree of the magnetic susceptibility in the 2-D case. The degree of anisotropy $P=K_{1} / K_{3}$ of a SD grain in a thermo-demagnetized state and in a AF-demagnetized state as a function of the interaction field $H$ computed for the parameter $a=0.3$. The shaded area is the area where the NRM is likely to be.

From here the maximum magnetic susceptibility along the direction $\psi=\pi / 2$ is:

$$
K_{1}=\frac{1}{4 \pi} \int_{0}^{\pi} \frac{2-a \cos \left(2 \theta_{0}(\phi)\right)}{F\left[\theta_{0}(\phi)\right]} d \phi
$$

Along the direction $\psi=0$, the minimum magnetic susceptibility is:

$$
K_{3}=\frac{1}{4 \pi} \int_{0}^{\pi} \frac{2+a \cos \left(2 \theta_{0}(\phi)\right)}{F\left[\theta_{0}(\phi)\right]} d \phi
$$

Using the same development for the AF-demagnetized state, we calculate the minimal and maximal magnetic susceptibility:

$$
K_{1}=\frac{1}{2 \pi} \int_{0}^{\frac{\pi}{2}} \frac{2-a \cos \left(2 \theta_{0}(\phi)\right)}{F} d \phi
$$

and

$$
K_{3}=\frac{1}{2 \pi} \int_{0}^{\frac{\pi}{2}} \frac{2+a \cos \left(2 \theta_{0}(\phi)\right)}{F} d \phi
$$

We find that the anisotropy degree $P=K_{1} / K_{3}$ decreases as the interaction field $H$ increases (Fig. 7). As a consequence, when interactions are relatively strong, the anisotropy ellipsoid is approaching a sphere, introducing noise in measurements. Let us investigate the 3-D geometry case, using the same approach.

\section{3-D geometry}

Now let consider a real 3-D spherical system ( $\theta$ the colatitude and $\lambda$ the longitude), leaving the assumption that all the vectors lie in the same plane. Notations are the same than in the 2-D geometry. In this system, the different vectors are:

$$
\mathbf{m}=m\left(\begin{array}{c}
\sin \theta_{2} \cos \lambda_{2} \\
\sin \theta_{2} \sin \lambda_{2} \\
\cos \theta_{2}
\end{array}\right) \quad \mathbf{H}_{\mathbf{i n t}}=H_{\text {int }}\left(\begin{array}{c}
\sin \theta_{3} \cos \lambda_{3} \\
\sin \theta_{3} \sin \lambda_{3} \\
\cos \theta_{3}
\end{array}\right) \quad \mathbf{h}_{\text {ext }}=h_{\text {ext }}\left(\begin{array}{c}
\sin \theta \cos \lambda \\
\sin \theta \sin \lambda \\
\cos \theta
\end{array}\right)
$$




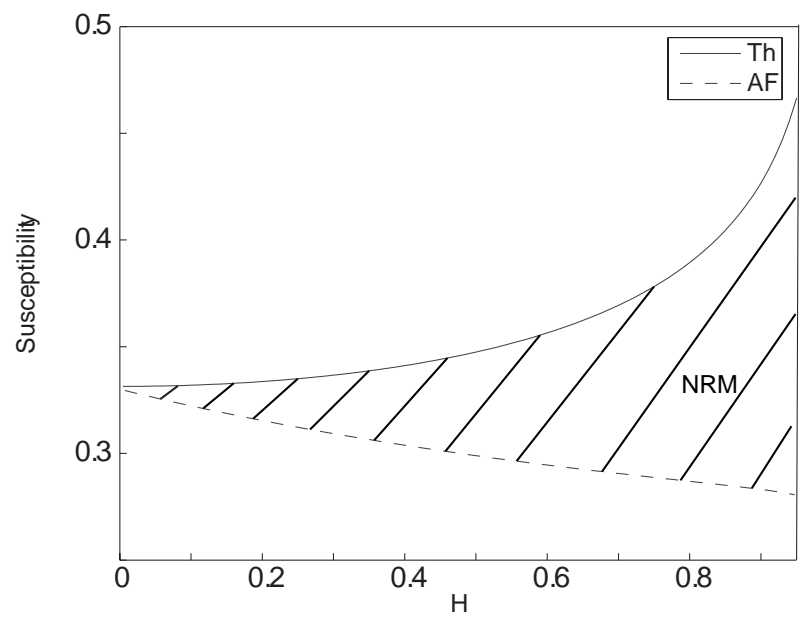

Figure 8: Influence of the interaction field on the bulk susceptibility in the 3D-case. Theoretical magnetic susceptibility of AF-demagnetized and thermo-demagnetized grain as a function of the interaction field $\mathrm{H}$. The shaded area is the area where the NRM is likely to be.

Without loss of generality and in order to simplify the problem, we assume that the easy axis of magnetization and the polar axis are aligned. This assumption implies that $\lambda_{3}=0, \theta_{2} \in[0 ; \pi]$, $\lambda_{2} \in[0 ; 2 \pi]$ and $\theta_{3} \in[0 ; \pi]$. The initial geometry is the same than in the $2 \mathrm{D}$ case. Now the un-perturbed part of the dimensionless energy transforms to:

$$
E_{0}=\sin ^{2} \theta_{2}-H\left(\sin \theta_{2} \sin \theta_{3} \cos \lambda_{2}+\cos \theta_{2} \cos \theta_{3}\right) .
$$

The stable points $\left(\theta_{2}^{0}, \lambda_{2}^{0}\right)$ can be found as roots of the equations:

$$
\frac{\partial E_{0}}{\partial \theta_{2}}=\sin \left(2 \theta_{2}\right)+H\left(\cos \theta_{3} \sin \theta_{2}-\cos \theta_{2} \sin \theta_{3} \cos \lambda_{2}\right)=0,
$$

and

$$
\frac{\partial E_{0}}{\partial \lambda_{2}}=H \sin \theta_{2} \sin \theta_{3} \sin \lambda_{2}=0 .
$$

From eq. (31), we obtain $\lambda_{2}=0$, for $H \neq 0, \theta_{2} \neq 0$ and $\theta_{3} \neq 0$. Then eq. (27) becomes:

$$
\frac{\partial E_{0}}{\partial \theta_{2}}=\sin \left(2 \theta_{2}\right)+H \sin \left(\theta_{2}-\theta_{3}\right)=0 .
$$

This equation is exactly the same as eq. (9) used for the 2D case. Let us look at perturbation of the energy caused by the applied small external field $\mathbf{h}_{\text {ext }}$. Under the action of this field, the coordinates of the equilibrium position $\left(\theta_{2}^{0}, 0\right)$ is slightly shifted to the position $\left(\theta_{2}^{0}+y, z\right)$ where $(y, z) \ll 1$. Using a Taylor's expansion around $\left(\theta_{2}^{0}, 0\right)$ :

$$
\begin{aligned}
E_{t o t}^{\prime}\left(\theta_{2}^{0}+y, z\right) & =\frac{y^{2}}{2} \frac{\partial^{2} E_{t o t}}{\partial \theta_{2}^{2}}\left(\theta_{2}^{0}, 0\right)+\frac{z^{2}}{2} \frac{\partial^{2} E_{t o t}}{\partial \lambda_{2}^{2}}\left(\theta_{2}^{0}, 0\right) \\
& +\frac{1}{2} \frac{\partial^{2} E_{t o t}}{\partial \theta_{2} \partial \lambda_{2}}\left(\theta_{2}^{0}, 0\right)-\left(\mathbf{h}_{\text {ext }} \cdot \mathbf{M}_{\mathbf{s}}\right) .
\end{aligned}
$$

where the vector of magnetization $\mathbf{M}_{\mathbf{s}}$ can be written as: 


$$
\mathbf{M}_{\mathbf{s}}=\left[\cos z \sin \left(\theta_{2}^{0}+y\right), \sin z \sin \left(\theta_{2}^{0}+y\right), \cos \left(\theta_{2}^{0}+y\right)\right] .
$$

The mixed derivative in eq. (34) is equal to zero because $\lambda_{2}^{0}=0$. Let $F$ and $G$ be the second derivatives of $E_{\text {tot }}$ over $\theta_{2}$ and over $\lambda_{2}$, respectively, defined by:

$$
F=\frac{\partial^{2} E_{t o t}}{\partial \theta_{2} 2}\left(\theta_{2}^{0}, 0\right)=2 \cos \left(2 \theta_{2}^{0}\right)+H\left(\cos \theta_{2}^{0} \cos \theta_{3}-\sin \theta_{2}^{0} \sin \theta_{3}\right)
$$

and

$$
G=\frac{\partial^{2} E_{t o t}}{\partial \lambda_{2}}\left(\theta_{2}^{0}, 0\right)=H \sin \theta_{2}^{0} \sin \theta_{3}
$$

Then, using these notations, eq. (34) becomes:

$$
\begin{aligned}
E_{t o t}\left(\theta_{2}^{0}+y, z\right) & \approx \frac{y^{2}}{2} F+\frac{z^{2}}{2} G-h \sin \left(\theta_{2}^{0}+y\right) \cos z \sin \theta \cos \lambda \\
& -h\left[\sin z \sin \left(\theta_{2}^{0}+y\right) \sin \theta \sin \lambda+\cos \left(\theta_{2}^{0}+y\right) \cos \theta\right]
\end{aligned}
$$

From here, recalling that $z \ll 1$ and $h \ll 1$, eq. (38) becomes:

$$
\begin{aligned}
E_{\text {tot }}\left(\theta_{2}^{0}+y, z\right) & \approx \frac{y^{2}}{2} F+\frac{z^{2}}{2} G \\
& -h\left[\sin \left(\theta_{2}^{0}+y\right) \cos z \sin \theta \cos \lambda+\sin z \sin \left(\theta_{2}^{0}+y\right) \sin \theta \sin \lambda+\cos \left(\theta_{2}^{0}+y\right) \cos \theta\right] .
\end{aligned}
$$

Moreover, at the stable position, we have:

$$
\frac{\partial E}{\partial y}=F y-h\left(\cos \theta_{2}^{0} \cos \lambda \sin \theta-\sin \theta_{2}^{0} \cos \theta\right)=0
$$

and

$$
\frac{\partial E}{\partial z}=G z-h\left(\sin \theta_{2}^{0} \sin \lambda \sin \theta\right)=0
$$

Solving the system, we find $y$ and $z$. Now, using these results and from eq. (35), the vector of the induced magnetization is:

$$
\mathbf{M}_{\mathbf{i n d}}=\left[y \cos \theta_{2}^{0}, z \sin \theta_{2}^{0},-y \sin \theta_{2}^{0}\right]
$$

The susceptibility is the projection of the induced magnetization $\mathbf{M}_{\mathbf{i n d}}$ on $\mathbf{h}$ :

$$
\begin{aligned}
\chi & =\mathbf{M}_{\mathbf{i n d}} \mathbf{h} / h^{2}=\frac{y}{h}\left[\cos \theta_{2}^{0} \sin \theta \cos \lambda-\sin \theta_{2}^{0} \cos \theta\right]+\frac{z}{h} \sin \theta_{2}^{0} \sin \theta \sin \lambda \\
& =\frac{1}{F}\left[\cos \theta_{2}^{0} \sin \theta \cos \lambda-\sin \theta_{2}^{0} \cos \theta\right]^{2}+\frac{1}{G}\left[\sin \theta_{2}^{0} \sin \theta \sin \lambda\right]^{2}
\end{aligned}
$$

For the thermal-demagnetized state with no magnetic fabric, the angles $\theta$ and $\lambda$ are randomly distributed over the sphere. Then, integrating eq. (43) over $\sin \theta d \theta d \lambda$, we obtain: 


$$
\begin{aligned}
\chi_{T} & =\frac{1}{8 \pi}\left(\int_{0}^{\pi} \frac{\cos ^{2} \theta_{2}^{0}}{F\left(\theta_{2}^{0}\right)} \sin \theta_{3} d \theta_{3} \int_{0}^{2 \pi} \int_{0}^{\pi}(\sin \theta \cos \lambda)^{2} \sin \theta d \theta d \lambda\right. \\
& +\int_{0}^{\pi} \frac{\sin ^{2} \theta_{2}^{0}}{F\left(\theta_{2}^{0}\right)} \sin \theta_{3} d \theta_{3} \int_{0}^{2 \pi} \int_{0}^{\pi} \cos ^{2} \theta \sin \theta d \theta d \lambda \\
& \left.+\int_{0}^{\pi} \frac{\sin ^{2} \theta_{2}^{0}}{G\left(\theta_{2}^{0}\right)} \sin \theta_{3} d \theta_{3} \int_{0}^{2 \pi} \int_{0}^{\pi}(\sin \theta \sin \lambda)^{2} \sin \theta d \theta d \lambda\right)
\end{aligned}
$$

After integrating on $\theta$ and $\lambda$ we have:

$$
\chi_{T}=\frac{1}{6}\left[\int_{0}^{\pi} \frac{\sin \theta_{3}}{F\left(\theta_{2}^{0}\right)} d \theta_{3}+\int_{0}^{\pi} \frac{\sin ^{2} \theta_{2}^{0}}{G\left(\theta_{2}^{0}\right)} \sin \theta_{3} d \theta_{3}\right]
$$

For the AF state, eq. (49) must be written:

$$
\chi_{A F}=\frac{1}{3}\left[\int_{0}^{\pi / 2} \frac{\sin \theta_{3}}{F\left(\theta_{2}^{0}\right)} d \theta_{3}+\int_{0}^{\pi / 2} \frac{\sin ^{2} \theta_{2}^{0}}{G\left(\theta_{2}^{0}\right)} \sin \theta_{3} d \theta_{3}\right]
$$

The plot of total susceptibility as a function of $H$ is represented in Fig. 8. In absence of interactions $(\mathrm{H}=0)$, we have $\theta_{2}^{0}=0$ and $\chi=1 / 3$. Indeed, the 3 - $\mathrm{D}$ problem can be split in three 1 -D problems. We consider one axis parallel to $\mathbf{h}_{\mathbf{e x t}}$, hence along this axis, the susceptibility is null. Then we consider the two other axes perpendicular to the first one. We have:

$$
\chi=\frac{M_{s}^{2}}{2 K} \sin ^{2}(\pi / 2)=\frac{J_{s}^{2}}{2 K}
$$

Averaging the susceptibility of these three directions we obtain:

$$
\chi=\frac{M_{s}^{2}}{3 K}
$$

This result is in agreement with the numerical integration (Fig. 8). As it is seen, these graphs are very similar to Fig. 5. So, all other of our conclusions on the role of interactions on the magnetic susceptibility are valid for the general 3-D ensemble.

If a magnetic fabric is present, by the analogy with the eq.(25) for the 2-D case, we can generalize the eq. (44) as follows:

$$
\begin{aligned}
\chi & =\frac{1}{8 \pi}\left(\int_{0}^{\pi} \frac{\cos ^{2} \theta_{2}^{0}}{F\left(\theta_{2}^{0}\right)} \sin \theta_{3} d \theta_{3} \int_{0}^{2 \pi} \int_{0}^{\pi}(\sin \theta \cos \lambda)^{2}[1+a \cos (2 \theta)] \sin \theta d \theta d \lambda\right. \\
& +\int_{0}^{\pi} \frac{\sin ^{2} \theta_{2}^{0}}{F\left(\theta_{2}^{0}\right)} \sin \theta_{3} d \theta_{3} \int_{0}^{2 \pi} \int_{0}^{\pi} \cos ^{2} \theta[1+a \cos (2 \theta)] \sin \theta d \theta d \lambda \\
& \left.+\int_{0}^{\pi} \frac{\sin ^{2} \theta_{2}^{0}}{G\left(\theta_{2}^{0}\right)} \sin \theta_{3} d \theta_{3} \int_{0}^{2 \pi} \int_{0}^{\pi}(\sin \theta \sin \lambda)^{2}[1+a \cos (2 \theta)] \sin \theta d \theta d \lambda\right)
\end{aligned}
$$

where the coefficient $a>0$ when the field is directed along the minimum susceptibility axis and vice versa. The integration of eq. 49 gives:

$$
\begin{aligned}
\chi_{T} & =\frac{1}{8 \pi}\left(\int_{0}^{\pi} \frac{\cos ^{2} \theta_{2}^{0}}{F\left(\theta_{2}^{0}\right)} \sin \theta_{3} d \theta_{3}(1 / 6+a / 10)+\int_{0}^{\pi} \frac{\sin ^{2} \theta_{2}^{0}}{F\left(\theta_{2}^{0}\right)} \sin \theta_{3} d \theta_{3}(1 / 6-a / 30)\right. \\
& +\int_{0}^{\pi} \frac{\sin ^{2} \theta_{2}^{0}}{G\left(\theta_{2}^{0}\right)} \sin \theta_{3} d \theta_{3}(1 / 6+a / 10)
\end{aligned}
$$




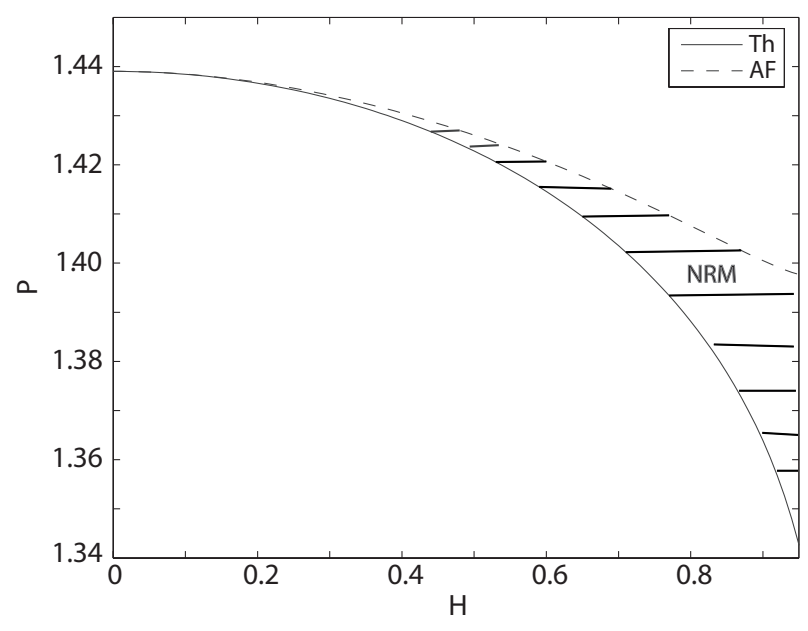

Figure 9: Influence of the interaction field on the anisotropy degree of the magnetic susceptibility in the 3-D case. The degree of anisotropy $P=K_{1} / K_{3}$ of a SD grain in a thermo-demagnetized state and in a AF-demagnetized state as a function of the interaction field $H$ computed for the parameter $a=0.3$. The shaded area is the area where the NRM is likely to be.

In the case of AF-state, we obtain:

$$
\begin{aligned}
\chi_{A F} & =\frac{1}{4 \pi}\left(\int_{0}^{\pi / 2} \frac{\cos ^{2} \theta_{2}^{0}}{F\left(\theta_{2}^{0}\right)} \sin \theta_{3} d \theta_{3}(1 / 6+a / 10)+\int_{0}^{\pi / 2} \frac{\sin ^{2} \theta_{2}^{0}}{F\left(\theta_{2}^{0}\right)} \sin \theta_{3} d \theta_{3}(1 / 6-a / 30)\right. \\
& +\int_{0}^{\pi / 2} \frac{\sin ^{2} \theta_{2}^{0}}{G\left(\theta_{2}^{0}\right)} \sin \theta_{3} d \theta_{3}(1 / 6+a / 10)
\end{aligned}
$$

As in the 2-D model, the degree of anisotropy decreases when the interaction field increases (Fig 9 ). The evolution of the degree of anisotropy $P$ as function of $H$ presents exactly the same trend in the 2-D and the 3-D geometry.

\section{Discussion and Conclusion}

Our model is quite different from the other numerical models dealing with AMS (Stephenson (1994), Cañon-Tapia (1996), Gaillot et al. (2006)) by its geometry. Indeed, all these models rely on a nonuniform distribution (lineation or foliation) of magnetic particles with a particular configuration of the interaction field. They predict that the degree of anisotropy is increased by magnetic interactions induced by the anisotropy of distribution. Here we have shown that it is not at all always the case, namely, random magnetic fields generated by the random arrangement of magnetic particles over the volume leads to the decrease of the degree of anisotropy. For this, a new method to estimate the role of interactions in an ensemble of randomly distributed SD grains over the volume is suggested.

However, it must be stressed that the degree of anisotropy depends on a number of other parameters, for example, AMS can be a result of a mixing of the interacting and non-interacting part fraction (Cañon-Tapia, 2001; Gaillot et al., 2006). The role of interactions between neighboring clusters is also questionable. Indeed, interactions between clusters can have also a contribution in the magnetic fabric. Depending on the distribution of the clusters and of their spacing, such interactions can raise an important magnetic fabric (Stephenson (1994), Cañón-Tapia (1996), Gaillot et al. (2006)), in opposition with the result expected according to our model. However, we showed that the interaction field is of the order of $100 \mathrm{kA} / \mathrm{m}$ and thus greater than the applied field during AMS measurements $(\approx 400 \mathrm{~A} / \mathrm{m}$ for the Kappabridge). Thus interactions are stronger within the clusters 
than between the clusters themselves and the influence of remote interactions may be neglected. Other sources of AMS can be present in the rocks such as shape orientation of non interacting magnetite grains. The AMS measurements do not allow to distinguish the main sources of AMS in the rocks. Thus it is important to identify the magnetic carriers and their properties to be able to interpret correctly magnetic fabrics and the rock history. Finally, the question concerning the roles of interactions in the remanent magnetization must be asked. Muxworthy and Williams (2004); Egli (2006b) have shown that densely packed interacting particles with uniaxial anisotropy also interfere with the remanent magnetization.

\section{Acknowledgement}

The stay of V. Shcherbakov in Montpellier Laboratory received the financial support from the CNRS and the Royal Academy of Sciences from Russia in the project 'Analysis and verification of paleointensity data obtained from territories of the former Soviet Union between the early 1960th up to the late 1990th'. We thank J. Jezek and an anonymous referee for their thorough reviews and constructive comments.

\section{References}

Bascou, J., Camps, P., Dautria, J. M., 2005. Magnetic versus crystallographic fabrics in a basaltic lava flow. J. Volcanol. Geotherm. Res. 145, 119-135.

Borradaile, G. J., Jackson, M., 2004. Anisotropy of magnetic susceptibility (AMS): magnetic petrofabrics of deformed rocks. In: F. Martìn-Hernàndez, C. M. Lüneburg, C. Aubourg and M. Jackson, Magnetic Fabrics: Methos and Applications. Geological Society, London.

Bouchez, J. L., 2000. Anisotropie de susceptibilité magnétique et fabriques des granites. C.R. Acad. Sci. Paris, Sciences de la Terre et des Planètes/ Earth and Planetary Sciences 330, 1-14.

Cañón-Tapia, E., 1996. Single-grain distribution anisotropy: A simple three-dimensional model. Phys. Earth Planet. Inter. 94, 117-131.

Cañon-Tapia, E., 2001. Factors affecting the relative importance of shape and distribution anisotropy in rocks: Theory and experiments. Tectonophysics 340, 117-131.

Cañón-Tapia, E., 2004. Anistropy of magnetic susceptibility in lava flows and dikes: an historical account. In: F. Martìn-Hernàndez, C. M. Lüneburg, C. Aubourg and M. Jackson, Magnetic Fabrics: Methos and Applications. Geological Spciety, London.

Chen, A. P., Egli, R., Moskowitz, B. M., 2007. First-order reversal curve (FORC) diagrams of natural and cultured biogenic magnetic particles. J. Geophys. Res. 112, doi:10.1029/2006JB004575.

Dunlop, D. J., Özdemir, O., 1997. Rock Magnetism: Fundamentals and frontiers. Cambridge University Press.

Egli, R., 2006a. Theoretical aspects of dipolar interactions and their appearance in first-order reversal curves thermally activated. J. Geophys. Res. 111, B12S17, doi:10.1029/2006JB004567.

Egli, R., 2006b. Theoretical considerations on the anhysteretic remanent magnetization of interacting particles with uniaxial anisotropy. J. Geophys. Res. 111,B12S18, doi:10.1029/2006JB004577.

Fanjat, G., Camps, P., Shcherbakov, V., Barou, F., Sougrati, M. T., Perrin, M., 2011. Magnetic interactions at the origin of abnormal magnetic fabrics in lava flows: A case study from Kerguelen flood basalts. Geophys. J. Int. in review, submitted. 
Gaillot, P., de Saint Blanquat, M., Bouchez, J. L., 2006. Effects of magnetic interactions in anisotropy of magnetic susceptibility: Models, experiments and implications for igneous rock fabrics quantification. Tectonophysics 418, 3-19.

Grégoire, V., de Saint Blanquat, M., Nédélec, A., Bouchez, J., 1995. Shape anisotropy versus magnetic interactions of magnetite grains: Experiments and application to AMS in granitic rocks. Geophys. Res. Lett. 22, 2765-2768.

Grégoire, V., Derrozes, J., Gaillot, P., Nédé1ec, A., Launeau, P., 1998. Magnetite grain fabric and distribution anisotropy vs. rock magnetic fabric a three-dimensional case study. J. Struct. Geol. 20, 937-944.

Hargraves, R. B., Johnson, D., Chan, C., 1991. Distribution anisotropy: The cause of AMS igneous rocks? Geophys. Res. Lett. 18, 2193-2196.

Jezek, J., Hrouda, F., 2004. Determination of the orientation of magnetic minerals from the anisotropy of magnetic susceptibility. Geological Society, London, Special Publications 238, 9-20.

Merle, O., 1998. Internal strain within lava flows from analogue modelling. J. Volcanol. Geotherm. Res. 81, 189-206.

Muxworthy, A., Williams, W., 2004. Distribution Anisotropy: The influence of magnetic interactions on the anisotropy of magnetic remanence. In: F. Martìn-Hernàndez, C. M. Lüneburg, C. Aubourg and M. Jackson, Magnetic Fabrics: Methos and Applications. Geological Society, London.

Nagata, T., 1961. Rock magnetism. Maruzen Co., Ltd.

O’Reilly, W., 1984. Rock and mineral magnetism. Blackie.

Potter, D. K., Stephenson, A., 1988. Single-domains particles in rocks and magnetic fabric analysis. Geophys. Res. Lett. 15, 1097-1100.

Rochette, P., Aubourg, C., Perrin, M., 1999. Is this magnetic fabric normal? A review and case studies in volcanic formations. Tectonophysics 307, 219-234.

Shcherbakov, V. P., Shcherbakova, V., 1975. On the magnetostatic interaction in a system of singledomain grains. Izv.-Phys. Solid Earth 9, 101- 104.

Stephenson, A., 1994. Distribution anisotropy: Two simple models for magnetic lineation and foliation. Phys. Earth Planet. Inter. 25, 49-53.

Tarling, D. H., Hrouda, F., 1993. The magnetic anistropy of rocks. Chapman and Hall, London.

Torquato, S., Truskett, T. M., Debenedetti, P. G., 2000. Is random close packing of spheres well defined? Phys. Rev. Lett. 81, 2064-2067.

Zhou, W., der Voo, R. V., Peacor, D. R., 1997. Single-domain and superparamagnetic titanomagnetite with variable $\mathrm{Ti}$ content in young ocean-floor basalts: No evidence for rapid alteration. Earth Plan. Sci. Lett. 150, 353-362. 